\title{
Optical System Design of Solar Blind Ultraviolet Imaging Detector
}

\author{
1Zhang Yunfei ${ }^{2}$ WuTian \\ 1. State Grid Jiangsu Electric Power Company, JiangSu, Xuzhou, 221000 \\ 2. RayieeTechnology(Beijing)CompanyLimited ,Beijing,100055
}

\begin{abstract}
Solar blind ultraviolet imaging detector is useful in detecting corona of overhead electric transmission lines, reducing power equipment damage. UV lens are the core of solar blind ultraviolet imaging detector. Their property affects the performance of the whole detector directly. In view of the problems of insufficient light energy utilization in corona detection system, the paper proposes a coaxial optical system based on wavelength and light separation, which eliminates the center field, enlarges the aperture of UV objective, and effectively improves the energy gathering ability of corona detection system. The mean square of the designed UV objective full view and whole detection range is less than $0.14 \mathrm{~mm}$, meeting the requirement of ultraviolet corona detection resolution. Compared with foreign ultraviolet optical system imaging detectors, the proposed solar blind ultraviolet imaging detector with coaxial optical system has better energy collection efficiency.
\end{abstract}

\section{INTRODUCTION}

The physics effect of cosmic rays and formation radioactive material make a small amount of charged ions in the air. The charged ions do directional movement under the action of electric field which forms electrical current. When the electric field intensity increases between transmission equipment to a certain value (generally between $20 \sim 30 \mathrm{kv} / \mathrm{cm}$ ), the charged ions in the air under the action of electric field get enough energy and make collision ionization with other molecular in the air. A channel with high conductivity is formed, which makes air local breakdown on the surface of high voltage transmission equipment and produces corona discharge [1-4]. Corona discharge can cause singeing, insulator corrosion, power line damage phenomena on transmission equipment surface, and more seriously it may cause power supply accidents. In addition, corona discharge is always with high frequency pulse electromagnetic wave radiation, so it will produce very big interference to radio communications. Therefore, corona blind UV imaging detector, which can make early detection and accurate positioning, is more and more brought to the attention of power sector [5-7]. Solar blind UV imaging detector through field height matches ultraviolet and visible light two channels, and make real-time acquisition of field UV and visible light image. Among them, UV channel works from $240 \mathrm{~nm}$ to $280 \mathrm{~nm}$ solar blind ultraviolet spectrum. Owing to the strong absorption of ozone in the stratosphere, the spectrum radiation in solar spectrum near the ground is almost non-existent in the atmosphere, so the spectral imaging detection can naturally avoid nature complex background interference. Solar blind UV imaging detector achieves corona early imaging detection making use of the characteristics of blind ultraviolet light contained in corona radiation spectrum. As early as the late $1970 \mathrm{~s}$, foreign countries have carried out the study of ultraviolet radiation light signals. In the late $1990 \mathrm{~s}$, overseas researchers have embarked on solar blind UV imaging detector work, and then they were gradually applied to the market [8-10]. At present, the representative main products include Israel Ofil Company's SuperB, Luminar, and South Africa UViRCO's CoroCAM series and so on, which is shown in Figure 1. 


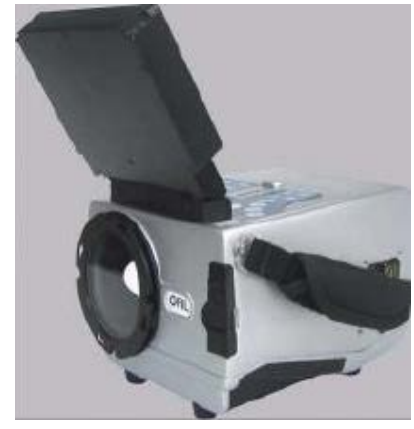

(a) SuperB

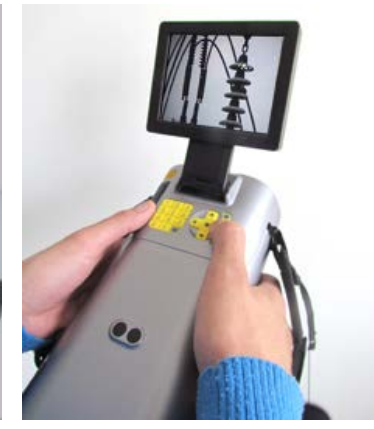

(b) Luminar

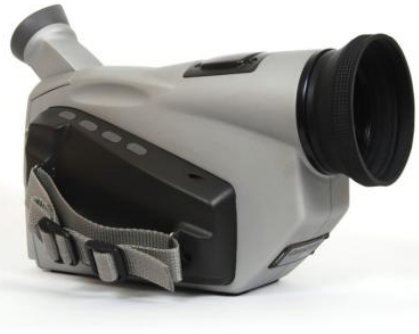

(c) CoroCAM

Figure 1 Typical UV imaging detector products abroad

Although the mentioned above products are of high sensitivity, positioning accuracy, simple operation, and low power consumption, there are many shortcomings. Its ultraviolet system structure is one of the typical faults. UV lens, as blind UV imaging detector core components, mainly affect parameters like optical aperture, light energy utilization, resolution of the detector and field, and its performance directly affects the performance of the detector. Cassegrain system is widely used in Ofil and UViRCO company products' ultraviolet optical lens. The center view exists fences, which lead to detector light diameter decrease and insufficient light energy utilization, as shown in Figure 2. This directly causes that when detector is telecommuting, in order to make the probe light energy meet imaging demand, usually require larger primary mirror size. In the aspect of application, the defect is not conducive to products miniaturization development.

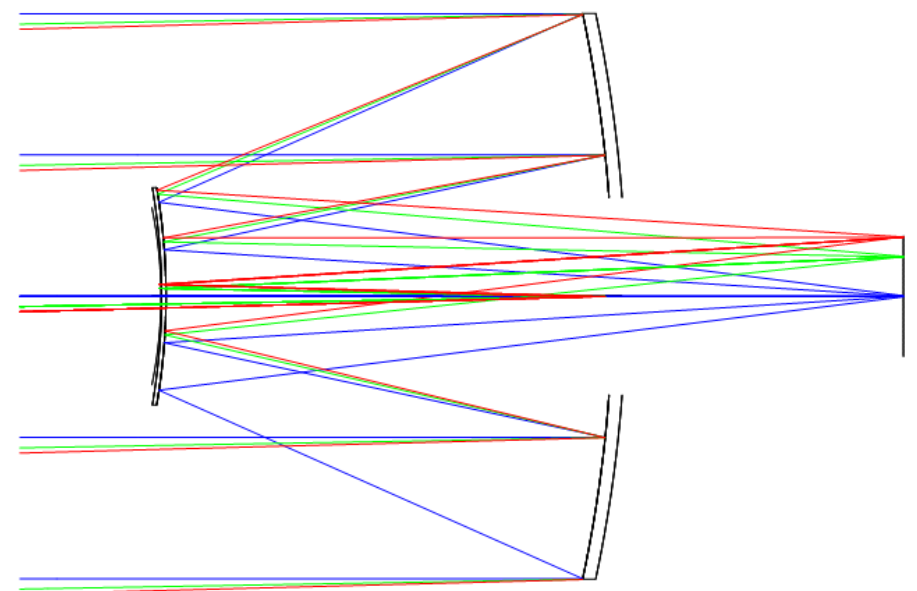

Figure 2 Cassegrain system schematic diagrams

Aiming at these problems, the article designs a kind of coaxial optical system based on color separation spectrophotometry, which eliminates center field fences, increases UV channel optical aperture, and effectively improves the ability of corona detection system energy collection. It provides practical reference for solar blind ultraviolet imaging detectors’ wide application.

\section{THE DETERMINATION OF OPTICAL SYSTEM PARAMETERS}

The technical indicators of solar blind ultraviolet imaging detectors are usually shown in the table below:

\begin{tabular}{lll}
\hline Detection distance & Angular resolution & View Angle \\
\hline $3 \mathrm{~m} \sim$ infinity & $\leq 0.1^{\circ}$ & $\geq 6^{\circ}$ \\
\hline
\end{tabular}

This system adopts UV ICCD diagonal size to $18 \mathrm{~mm}$. Because the "solar blind" ultraviolet light from corona discharge is extremely weak, provide greater incident luminous flux to UV ICCD than 
or equal to $1 \times 10-17 \mathrm{~W}$ to make UV ICCD light energy of incident satisfy the need of imaging. Target emitted radiation incidence after UV lens and filters to UV ICCD radiation flux can be calculated by the following formula:

$$
\Psi=\int_{240}^{280} k \frac{\pi D^{2}}{4} \frac{I(\lambda)}{L^{2}} \tau_{\text {filter }}(\lambda) \tau_{\text {lens }}(\lambda) \tau(\lambda)^{L / 1000} d \lambda
$$

In it, D is UV objective light aperture, $\mathrm{L}$ is the distance of the target to ultraviolet lens first surface, $\mathrm{k}$ is the related detection Angle coefficient, $\tau$ filter and $\tau$ lens are filter and UV objective transmittance respectively, $\tau(\lambda) \mathrm{L} / 1000$ is atmospheric attenuation coefficient.

In this system, the half width of UV band pass filter transmittance is about 0.21 , the ultraviolet lens overall transmittance designed is greater than or equal to 0.8 . Through the design requirements' view Angle indicators, the normal Angle between actual detection objects and the detector is small, and can be thought as $\mathrm{k}=1$ when calculating.

When parameter $\mathrm{k}=1$, $\operatorname{plug} \tau_{\text {filter }}=0.21$, and $\tau_{\text {lens }} \quad=0.8$ to the type, we can get:

$$
\Psi=\frac{\pi D^{2}}{4} \times 0.21 \times 0.8 \times \int_{240}^{280} \frac{I(\lambda)}{L^{2}} \tau(\lambda)^{L / 1000} d \lambda
$$

When detected minimal ultraviolet sensitivity is $3 \times 10$ to $18 \mathrm{w} / \mathrm{cm} 2$, combine with the above mentioned UV ICCD flux demand $10^{-17} \mathrm{~W}$; ultraviolet lens optical aperture size meeting the demand of imaging can be calculated:

\section{$\mathrm{D} \geq 50.215 \mathrm{~mm}$}

In order to ensure the quality of imaging, at the same time, consider the size space and mechanical structure, and the final design of diameter size is $52 \mathrm{~mm}$.

UV lens focal length can be got through the size of UV ICCD and system's optical field Angle calculation, which is shown as follows:

$$
f^{\prime}=\frac{d}{\tan 2 \omega^{\prime}}
$$

In it, $\mathrm{d}$ is ICCD diagonal dimension, and $2 \mathrm{w}$ ' is system optical viewing angle.

The ultraviolet lens focal length calculated is $\mathrm{f}^{\prime} \leq 171.73 \mathrm{~mm}$. From comprehensive consideration, finally the selected system focal length is $160 \mathrm{~mm}$.

In corona detection system, optical channel is used to detect the background image, and the image channel hope to keep the details of background image as much as possible. The "solar blind" UV channel is only used to detect whether there is phenomenon of corona discharge, and the image characteristics are mottled corona discharge area and no specific information in morphology of temporal images, so "solar blind" UV channel imaging does not care about content details, and concerns only light energy as well as imaging resolution. When $80 \%$ imaging dispersion spots in two corona discharge points do not overlap, the subsequent image processing system can separate two points through simple image processing algorithm. By system angular resolution requirements and system focal length, calculated $80 \%$ UV objective imaging dispersion spot radius $\mathrm{r}$ meet:

$r \leq \frac{f^{\prime} \times \Delta \theta}{2}$

Input the designed ultraviolet lens focal length and angular resolution demand, $r$ is less than or equal to $0.14 \mathrm{~mm}$.

To sum up, the designed UV lens specifications are as follows:

Optical aperture $52 \mathrm{~mm}$

Optical acuity field Angle $\geq 6^{\circ}$ 


\begin{tabular}{ll}
\hline Detection range & $3 \mathrm{~m} \sim$ infinity \\
\hline Working band & $240-280 \mathrm{~nm}$ \\
\hline System focal length & $160 \mathrm{~mm}$ \\
\hline Dispersion spot radius & $\leq 0.14 \mathrm{~mm}$ \\
\hline
\end{tabular}

\section{UV LENS DESIGN}

Optical materials transmittance mostly decreases with work wavelength reduction, thus lens material can be used to work for solar blind ultraviolet band is less. Commonly used materials are fused quartz and calcium fluoride. For calcium fluoride materials, the prices are more expensive and are in $240 \sim 280 \mathrm{~nm}$ wavelength range. Calcium fluoride shows stronger intrinsic birefringence, which seriously affects the resolution of the system [23]. Due to narrower actual working band, color difference is small, so the system uses fused quartz in the design as lens material. Due to "solar blind" ultraviolet light from corona discharge energy is weak, in order to avoid too many lenses leading to excessive energy loss and saving the cost of system at the same time, use less lens as far as possible when design to meet the system requirements, so adopt the method of splitting monolithic lenses for UV lens design. The designed steps are shown in the Figure below.

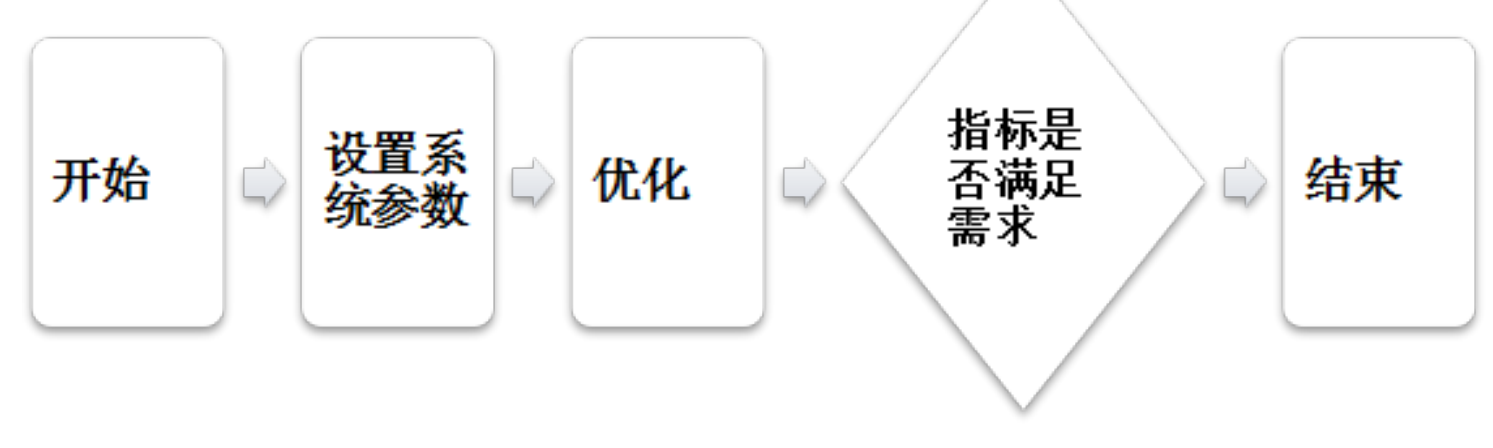

The final design results are follows:

\begin{tabular}{|c|c|c|c|c|c|c|c|}
\hline & Surf: Type & Comment & Radius & Thickness & Glass & Semi-Diameter & Conic \\
\hline $\mathrm{OBJ}$ & Standard & & Infinity & Infinity & & Infinity & 0.000000 \\
\hline 1 & Standard & & Infinity & 50.000000 & & 43.294567 & 0.000000 \\
\hline 2 & Coordinate B. & & & 0.000000 & - & 0.000000 & \\
\hline $3 \#$ & Standard & & Infinity & -200.000000 & MIRROR & $\begin{array}{ll}60.000000 & \text { U } \\
\end{array}$ & 0.000000 \\
\hline 4 & Coordinate B.. & & & 0.000000 & - & 0.000000 & \\
\hline $5 \#$ & Standard & & Infinity & 80.000000 & MIRROR & \begin{tabular}{ll|l}
50.000000 & $\mathrm{U}$ \\
\end{tabular} & 0.000000 \\
\hline $510 *$ & Standard & & 139.563741 & 20.272840 & F_SILICA & \begin{tabular}{|l|l}
27.000000 & U \\
\end{tabular} & 0.000000 \\
\hline $7 *$ & Standard & & Infinity & 8.340345 & & $\begin{array}{lll}27.000000 & \text { U } \\
\end{array}$ & 0.000000 \\
\hline $8 *$ & Standard & & 169.895708 & 12.404471 & F_SILICA & \begin{tabular}{|l|l}
27.000000 & U \\
\end{tabular} & 0.000000 \\
\hline $9^{*}$ & Standard & & $1.548053 \mathrm{E}+004$ & 6.379813 & & $27.000000 \mathrm{U}$ & 0.000000 \\
\hline $10 *$ & Standard & & -416.108899 & 12.389301 & $F_{-}$SILICA & \begin{tabular}{|l|l}
27.000000 & U \\
\end{tabular} & 0.000000 \\
\hline $11 *$ & Standard & & 2707.931131 & 160.371999 & & $27.000000 \mathrm{U}$ & 0.000000 \\
\hline 12 & Standard & & Infinity & 12.500000 & $F_{-}$SILICA & 11.759993 & 0.000000 \\
\hline 13 & Standard & & Infinity & 0.000000 & & 11.354303 & 0.000000 \\
\hline IMA & Standard & & Infinity & - & & 11.354303 & 0.000000 \\
\hline
\end{tabular}

System structure diagram is as follows: 


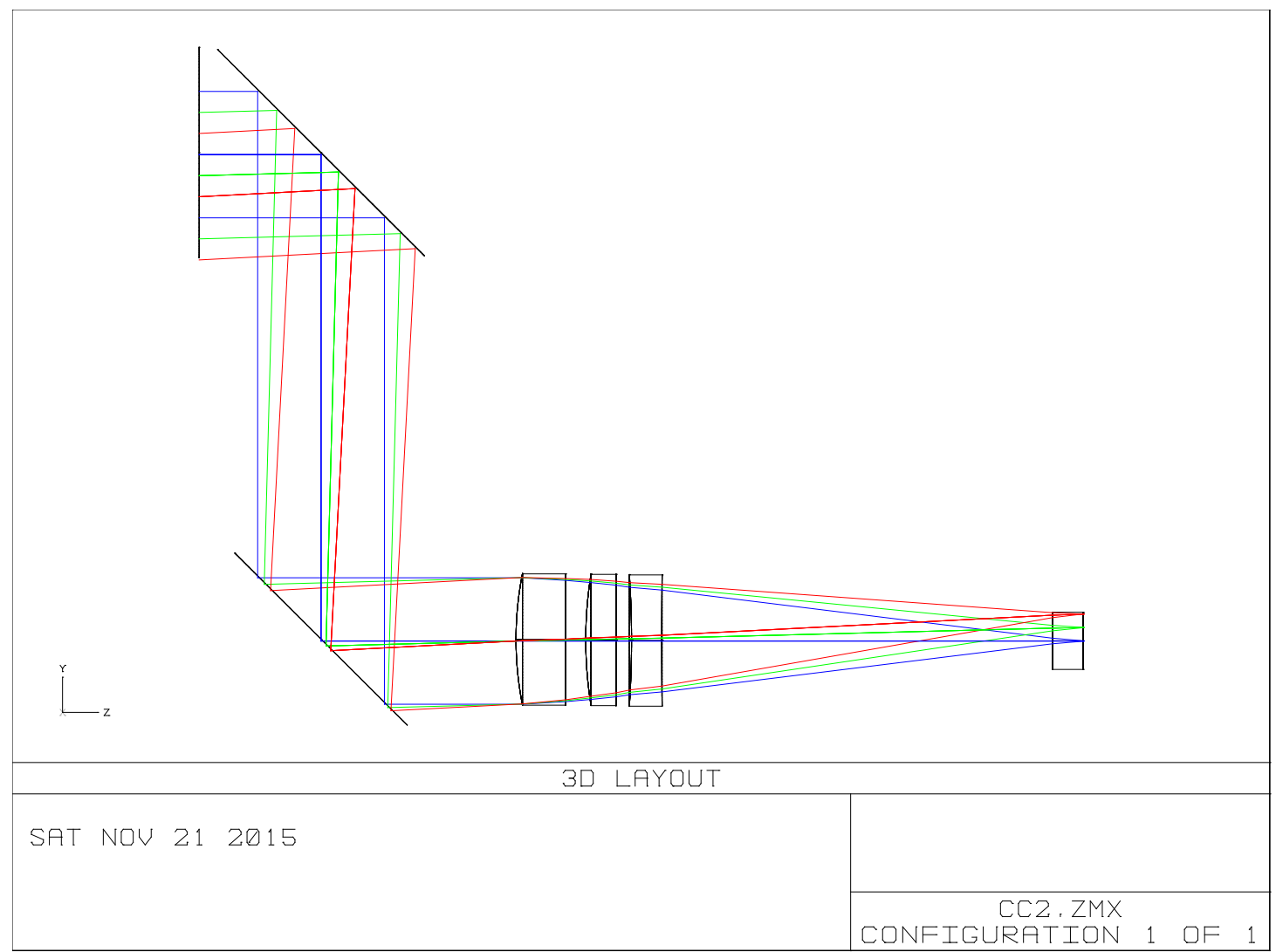

In the diagram, 1 reflector is spectral dichroic mirror surface, which is used to divide visible and ultraviolet light emitted from the target, and achieves UV-vis double path detection. Planes 12 and 13 are ultraviolet band pass filters, and UV ICCD image plane overlaps 13 planes. At the same time, ultraviolet lens can move as a whole through mechanical focusing structure to implement the target detection from $3 \mathrm{~m}$ to infinity.

\section{UV OBJECTIVE ANALYSIS}

A. Ultraviolet image quality evaluation:

In corona detection system, optical system of UV channel is the system for target signal energy detection, and the energy concentration of the system is the key index of evaluation system image quality [24]. In aberration system, column point's distribution can represent energy distribution, and the intensity of points can measure the merits of the system imaging quality. So energy detection system often uses column points as evaluation standard for the system.

The below diagram is each field's imaging point series when detection range is $3 \mathrm{~m}$ and infinity respectively. It can be seen from the diagram that in the whole detection range, the system's each field dispersion root mean square radius is less than $0.14 \mathrm{~mm}$, which meets the design requirements. 


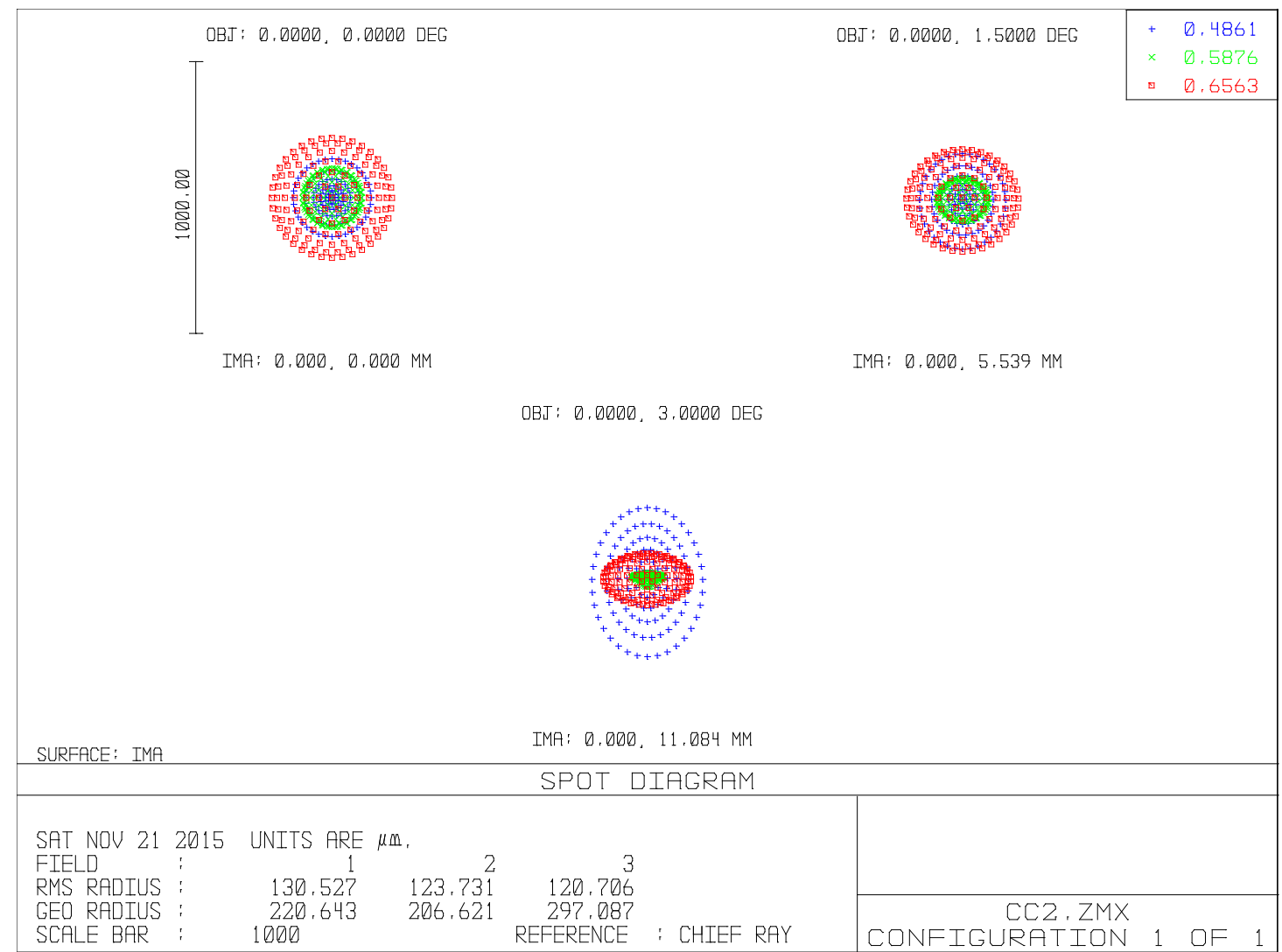

$3 \mathrm{~m}$

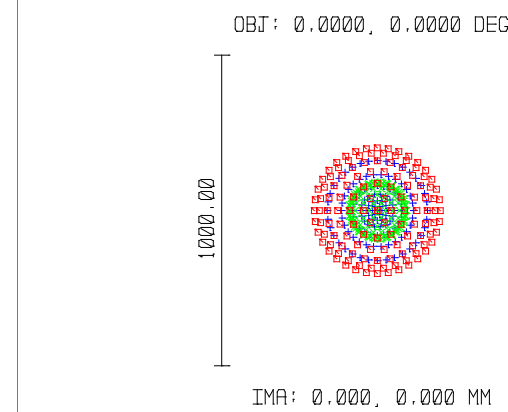

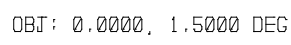
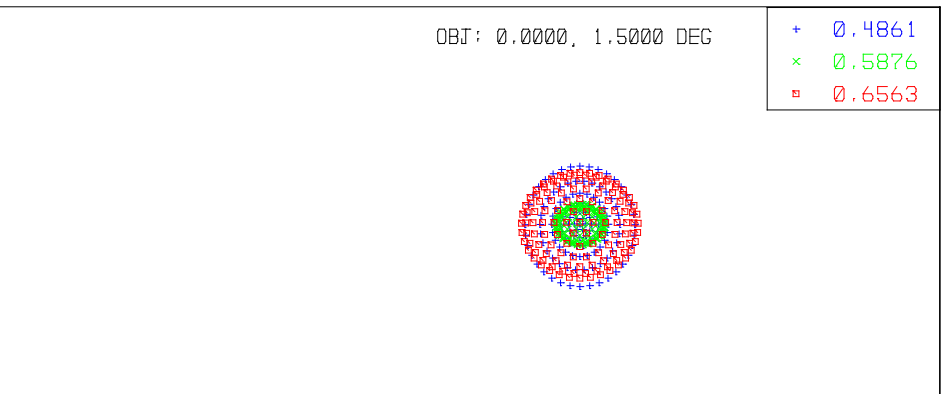

\begin{tabular}{rr}
$\times$ & 0.5876 \\
0 & 0.6563 \\
\hline
\end{tabular}

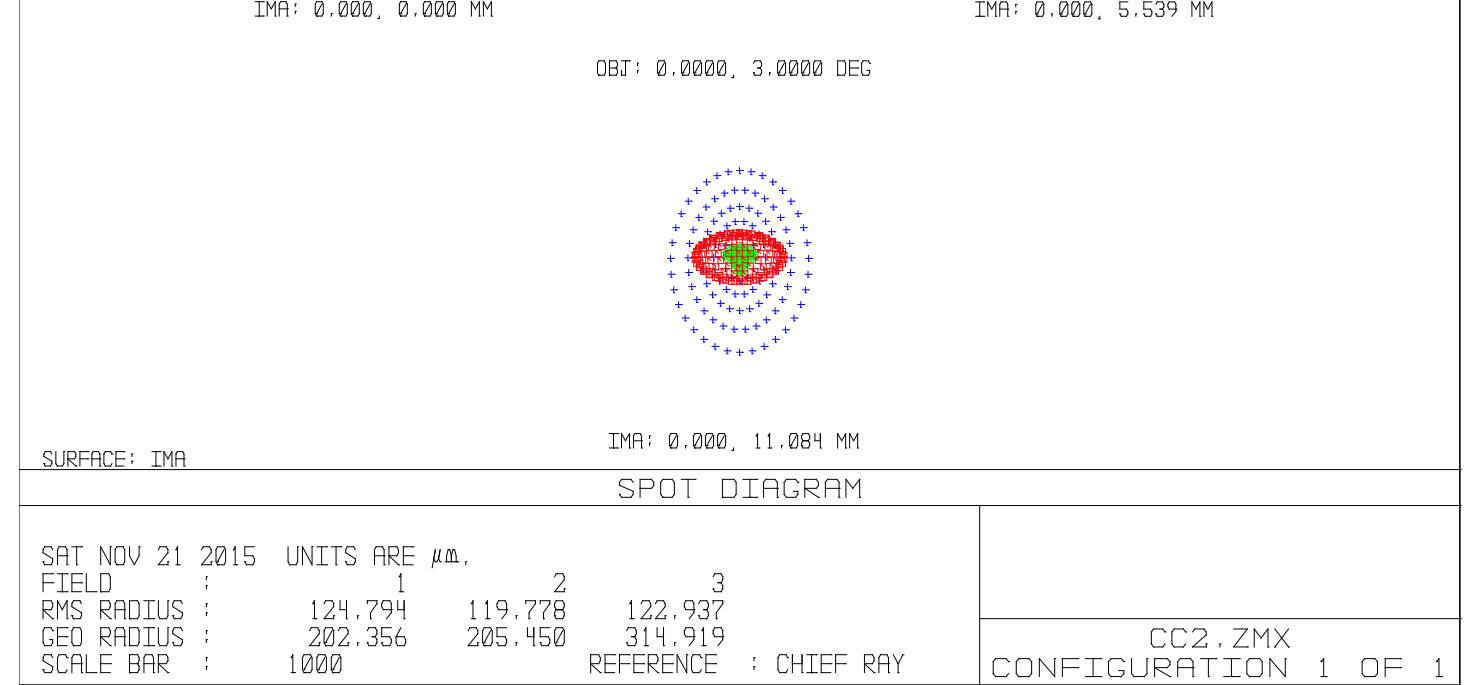

\section{Infinity}

\section{B. UV lens tolerance}

There are optical element processing and errors between practical optical system design and theoretically designed optical system, which change and reduce the actual performance and image quality evaluation only reflects UV lens imaging quality in ideal theoretical design. So when the 
theory design of the optical system meets the requirements of technical indicators, make preliminary tolerance parameters according to manufacturing and assembly capacity, and make tolerance analysis. Through the tolerance analysis of system, find out system sensitivity of tolerance, strictly control influence tolerance, and at the same time, set tolerance compensation reasonably to improve the tolerance of the system. And ultimately determine tolerance parameters in the system, and provide guidance for the subsequent mechanical design.

Comprehensively consider optical lens production level and laboratory assembly capacity, and eventually make the tolerances. It is shown in the following table. Optical component tolerances refractive index is set as 0.0001 , and abbe number tolerance is $0.1 \%$.

\begin{tabular}{|c|c|c|c|c|c|c|c|}
\hline \multirow{2}{*}{$\begin{array}{l}\text { Su } \\
\text { rfa } \\
\text { ce }\end{array}$} & \multicolumn{4}{|c|}{ Components machining tolerance } & \multicolumn{3}{|c|}{ Assembly tolerances } \\
\hline & $\begin{array}{l}\text { Surface } \\
\text { shape } \\
\text { toleranc } \\
\text { e }(\lambda)\end{array}$ & $\begin{array}{l}\text { Radius } \\
\text { of } \\
\text { toleran } \\
\text { ce }(\mathbf{N})\end{array}$ & $\begin{array}{l}\text { Thicknes } \\
\text { s } \\
\text { tolerance } \\
\text { ( } \mathbf{m m})\end{array}$ & $\begin{array}{l}\text { Element } \\
\text { wedge } \\
(\mathbf{m m})\end{array}$ & $\begin{array}{l}\text { Spacing } \\
\text { tolerance } \\
(\mathbf{m m})\end{array}$ & $\begin{array}{c}\text { Eccentric } \\
\text { tolerance } \\
(\mathbf{m m})\end{array}$ & $\begin{array}{l}\text { Tilted } \\
\text { tolerance } \\
\left({ }^{\prime}\right)\end{array}$ \\
\hline 6 & 0.25 & 2 & 0.05 & 0.05 & & 0.05 & 2 \\
\hline 7 & 0.25 & 2 & & & 0.05 & & \\
\hline 8 & 0.25 & 2 & 0.05 & 0.05 & & 0.05 & 2 \\
\hline 9 & 0.25 & 2 & & & 0.05 & & \\
\hline 10 & 0.25 & 2 & 0.05 & 0.05 & & 0.05 & 2 \\
\hline 11 & 0.25 & 2 & & & 焦距调节 & & \\
\hline
\end{tabular}

Use Monte Carlo simulation method for tolerance sensitivity analysis, and finally the results are as follows:

Point series figure root mean square 0.115735888

radius optimal value $(\mathbf{m m})$

Column chart root mean square radius 0.115766229

worst point $(\mathbf{m m})$

Point column figure root mean square 0.115749666

radius average $(\mathbf{m m})$

90\% mean square root radius maximum 0.115763594

( $\mathbf{m m}$ )

Former focal length offsets ( $\mathbf{m m}) \quad 0.115301 \mathrm{~mm}$

Latter focal length offsets ( $\mathrm{mm}$ ) $0.066264 \mathrm{~mm}$

It can be seen that $90 \%$ of the shots are able to meet the design requirements in the final set within the limits of tolerance, therefore, UV lens can be machinated and assembled based on the tolerance. Compared with foreign reflection type design, transmission design has more advantages on light energy utilization.

\begin{tabular}{cc}
\hline & Light energy utilization \\
\hline Reflection type & $71 \%$ \\
\hline Transmission type & $80 \%$ \\
\hline
\end{tabular}

\section{CONCLUSION}

This paper analyzes corona detection demands, puts forward the general design demand of 
ultraviolet lens, and designs UV lens applied to testing system through splitting method. Within $6^{\circ}$ field, the designed UV lens's point column figure mean square root are less than $0.14 \mathrm{~mm}$ when imaging range is from $3 \mathrm{~m}$ to infinity, which meets the demand resolution of ultraviolet corona detection. At the same time, combining with laboratory existing processing and assembling ability, tolerance analysis of the whole optical system is given. This paper's design of lens can meet the application demand of ultraviolet corona detection.

\section{REFERENCES}

[1] Zhang Haifeng, Pang Qichang, Chen Xiuchun. High voltage corona features and detection [J]. Electric Measurement and Instrumentation, 2006 lancet (2) : 6-8

[2] Wang Jingang. High voltage equipment discharge ultraviolet detection technology and its application research [D]. PhD Thesis, Chongqing University, 2008

[3] Gu Leguan, Sun Caixin. The pollution insulation of power system [M]. Chongqing: Chongqing University Press, 1990,59-61

[4] Xiao Dongping, He Wei, Xie Pengju etc. High voltage transmission line corona discharge characteristics and electromagnetic radiation field [J]. Power Grid Technology, 2007, 31 (21) : 52-55

[5] Dong Yongchao. Uhv transmission line corona discharge online monitoring system research [D]. Master's Degree Thesis, Control Theory and Control Engineering, Jiangsu University of Science and Technology, 2012

[6] Yan Feng, Yu Zijiang, Yu Xiao. Corona detection UV ICCD camera image noise analysis and process [J]. Journal of Optics, Precision Engineering, 2006, 14 (4) : 709-713

[7] Jungang Liu, Wei He, Liang Zhou, et al. Application of ultraviolet detector in discharge detection[J]. International Journal of Emerging Electric Power System, 2006, 7(3): Article 2

[8] Jin Pinggui. Ultraviolet detection technology and double spectrum image detection system research [D]. PhD Thesis, Xi 'an Optical Precision Machinery Research Institute, Chinese Academy of Sciences, 2004.

[9] Yan Feng, Wang Xin. Using real time embedded system with multiple DSPs in corona detection[J]. Proc of SPIE, 2006, 6027: 928-933.

[10] Zhang Mingchao, Song Huiying, Zhou Yue. Blind ultraviolet corona detecting system calibration [J]. Journal of Electrical Engineering, 2010, 5 (4) : 135-140 